\title{
BMJ Open Cross-sectional study examining the status of intrinsic capacity decline in community-dwelling older adults in China: prevalence, associated factors and implications for clinical care
}

\author{
Lina Ma (D) , ${ }^{1}$ Jagadish K Chhetri, ${ }^{1}$ Li Zhang, ${ }^{1}$ Fei Sun, ${ }^{2}$ Yun Li, ${ }^{1}$ Zhe Tang ${ }^{2}$
}

To cite: Ma L, Chhetri JK, Zhang L, et al. Cross-sectional study examining the status of intrinsic capacity decline in community-dwelling older adults in China: prevalence, associated factors and implications for clinical care. BMJ Open 2021;11:e043062. doi:10.1136/ bmjopen-2020-043062

- Prepublication history and additional materials for this paper is available online. To view these files, please visit the journal online (http://dx.doi. org/10.1136/bmjopen-2020043062).

Received 23 July 2020

Revised 07 December 2020 Accepted 16 December 2020

Check for updates

(C) Author(s) (or their employer(s)) 2021. Re-use permitted under CC BY-NC. No commercial re-use. See rights and permissions. Published by BMJ.

'Department of Geriatrics, Xuanwu Hospital, Capital Medical University, China National Clinical Research Center for Geriatric Disorders, Beijing, China

${ }^{2}$ Beijing Institute of Geriatrics, Xuanwu Hospital, Capital Medical University, Beijing, China

\section{Correspondence to}

Dr Lina Ma;

malina0883@126.com and

Dr Zhe Tang;

tangzhe@sina.com

\section{ABSTRACT}

Objectives Intrinsic capacity (IC) was proposed by the WHO as a new concept for capturing an individual's functional capacities across their lifetime. We aimed to investigate the prevalence and factors associated with IC decline and examine associations between IC and adverse outcomes among community-dwelling older adults in China.

Design A cross-sectional study.

Setting Community, China.

Participants Data were derived from the China Comprehensive Geriatric Assessment Study, a populationbased nationally representative sample. IC comprises of five domains: locomotion, cognition, vitality, sensory and psychology. Participants were deemed to have IC decline if they showed a decline in any of the five domains. Sociodemographic characteristics, chronic diseases, geriatric syndromes and adverse outcomes were also examined.

Results Of the 5823 community-dwelling participants aged 60-98 years, 2506 had IC decline (weighted $39.9 \%$ ): $57.7 \%$ in western, $38.3 \%$ in northern, $33.7 \%$ in northwest, $36.1 \%$ in middle, $16.9 \%$ in eastern and $19.8 \%$ in northeast China. The number of participants with decline in the locomotion, cognition, vitality, sensory and psychological domains were 1039 (17.8\%), $646(11.1 \%)$, 735 (12.6\%), $824(14.2 \%)$ and $713(12.2 \%)$, respectively. Age, northern residence, low education, being unmarried, low income, less exercise, less meat intake, insomnia, memory loss, urinary incontinence, constipation, slowness, chronic obstructive pulmonary disease and osteoarthritis were related to IC decline. After adjusting for age, sex, area, district, marriage, education, waist-hip ratio, smoking, alcohol consumption, exercise, income and chronic diseases, IC decline was independently associated with risk of frailty, disability, falls, fractures and immobility.

Conclusion The prevalence of IC decline in China is high. IC decline was significantly associated with adverse outcomes, after adjustment for related variables. Efforts promoting IC to delay functional dependence should focus on modifiable factors, including negative social factors, poor lifestyle, chronic diseases and geriatric syndromes.

\section{Strengths and limitations of this study}

- This study is the first nationally representative large sample of population-based older people focusing on intrinsic capacity (IC) in China.

- Most of the domains of IC were assessed using unified measured performance tests.

- This study provided a preliminary understanding of the IC status in China, which suggests ways to successfully put IC into clinical practice contributing to the development of integrated care strategies for older persons with IC decline.

- The cross-sectional design of this study limits causality.

- We used a composite total score instead of a weighted score, so further statistical approaches should be conducted to compute the IC score.

\section{INTRODUCTION}

The increasing global burden of disability associated with the rapid ageing of the population has challenged the healthcare system. ${ }^{1}$ To cope with the demands of increasing numbers of frail community-dwelling older persons with multiple complex needs, the implementation of integrated care healthcare model is necessary. ${ }^{2}$ The WHO has proposed an innovative approach known as the Integrated Care for Older People (ICOPE). This approach centres around the concept of raising intrinsic capacity (IC) and functional ability to achieve healthy ageing. Recommendations for the development of comprehensive approaches for the maintenance of IC and functional ability, including quality integrated care, have also been made. Moreover, the importance of integrated care in improving quality of life in the older population has been recently highlighted, ${ }^{3}{ }^{4}$ including reducing frailty in community-dwelling older people. 
According to the WHO ICOPE guidelines, IC comprises an individual's physical and mental capacities at any given time. ${ }^{4}$ This novel concept of IC has shifted the notion of 'healthy ageing' from a disease-centred to a functioncentred paradigm, creating opportunities for earlier intervention to delay disability and care dependency. The WHO proposes five components for the assessment of IC, namely, locomotion, cognition, vitality, sensory and psychology. ${ }^{3}$ Since each component is associated with adverse health events in older people, recent studies have focused on these components in combination. ${ }^{6-16}$ As so, our in-depth understanding of the trajectory of IC decline may enable us to design innovative methods for the promotion of healthy ageing, ${ }^{17}$ even during a catastrophic event such as the COVID-19. ${ }^{18}$ Although the concept of IC has been used in several studies worldwide, ${ }^{12} 1516$ no studies have been conducted in China. Therefore, to facilitate the evaluation of integrated care approaches for older patients, this study focuses on (1) the prevalence of IC decline and the factors associated with IC decline and (2) the associations between IC and geriatric conditions such as frailty, disability, falls, fractures and immobility in a population-based nationally representative sample of community-dwelling older people in China.

\section{METHODS}

\section{Participants}

Data were obtained from the China Comprehensive Geriatric Assessment Study (CCGAS, 2011-2012), a population-based face-to-face survey of adults aged 60 years or over from rural and urban areas of China. Further details regarding the CCGAS have been reported. ${ }^{19-21}$ For the CCGAS, seven cities (Beijing, Xi'an, Harbin, Chengdu, Chongqing, Changsha and Shanghai) from seven different provinces were chosen based on wellestablished cluster, stratification and random selection statistical sampling techniques. These cities represent China's six main regions (northern, middle, eastern, northwestern, southwestern and northeastern). A total of 6867 older adults were included in the CCGAS. A total of 5823 participants with complete IC data were included in our current study and 1040 were excluded because of missing data. A comparison of included and excluded participants' characteristics is shown in online supplemental table S1. All participants provided informed consent and the study design was approved by the ethics review board of Xuanwu Hospital, Capital Medical University.

\section{Demographics, lifestyles and diseases}

Data included sociodemographic characteristics, anthropometric measurements, health status, personal habits and mental health. Medical conditions, including a selfreported history of chronic disease diagnosed by a doctor, and geriatric syndromes were recorded. Low income was defined as monthly income $<2000 ¥$, no exercise was defined as exercising for $<3$ hour/week, and meat intake was measured by asking participants if they had meat diet $\geq 2$ times per week.

\section{IC construct}

IC was assessed using the five components recommended by the $\mathrm{WHO}^{322}$ as described below:

1. Locomotion: it was assessed by a short physical performance battery (SPPB), which comprised of various tests. ${ }^{23}$ The balance test comprised three parts: standing unsupported for $10 \mathrm{~s}$ with feet together; a semitandem stand and a full-tandem stand. Participants who were able to complete each part received a score of 1 ; otherwise, they were scored 0 . The chair-stand test was performed with the participant seated in a chair, with feet flat on the floor and arms held flat against their sides with elbows at $90^{\circ}$. Participants who were able to stand up five times from the chair received a score of 1 , those who partially completed this task scored 0.5, and those who could not stand up at all scored 0. A 20-metre walking test was also conducted: those who were able to walk $20 \mathrm{~m}$ scored 1 , those who walked less than $20 \mathrm{~m}$ scored 0.5 and those could not walk at all scored 0 . The total score range for the SPPB was $0-5$.

2. Cognition: it was assessed by the Mini-Mental State Examination, with a total score range of $0-30 .{ }^{24}$ The thresholds for participants who were illiterate or who were educated up to elementary school, middle school and high school or above were 17, 20 and 24, respectively. Participants who scored below the threshold value for their education group were regarded as mildly cognitively impaired (score of 1 ), and those who scored below 15 were regarded as moderately to severely cognitively impaired (score of 0 ).

3. Vitality: it was assessed by body mass index (BMI), which was calculated by dividing weight in kilograms by height in metres squared. BMI cutoffs were based on the Malnutrition Universal Screening Tool. ${ }^{25} \mathrm{~A}$ BMI above $20 \mathrm{~kg} / \mathrm{m}^{2}$ received a score of $2,18.5-20 \mathrm{~kg} / \mathrm{m}^{2}$ was scored 1 and $\leq 18.5 \mathrm{~kg} / \mathrm{m}^{2}$ was scored 0 .

4. Sensory: it was assessed by a self-reported visual and hearing impairment. Vision capacity was considered intact when the participant did not report 'eyesight problems' that interfered with their activities and were not identified by the interviewer as being functionally blind. Hearing capacity was considered intact when the participant did not report 'hearing problems or deafness' that interfered with their activities, and was not identified by the interviewer as being profoundly deaf. Participants with both visual and hearing impairments received a score of 0 , those with either vision or hearing impairment received a score of 1 , and those with intact vision and hearing capacities scored 2.

5. Psychology: it was measured by the 30-item Geriatric Depression Scale with a total score range of $0-30 .^{26}$ Scores from 0 to 10 represent intact psychological capacity (score of 2), scores from 11 to 20 represent mild 
depression (score of 1 ) and scores from 21 to 30 represent moderate to severe depression (score of 0 ).

For each of the five components, participants were scored either 0 (representing severe decline), 1 (representing mild decline) or 2 (representing intact IC). The total score of IC ranged from 0 to 10; higher scores indicated better IC.

\section{Adverse outcomes}

Physical function was assessed as activities of daily living (ADL) and instrumental ADL (IADL). The list of activities consisted of 14 items (eating, grooming, dressing, transferring in and out of bed, bathing, walking inside the house, using the toilet, cooking, managing finances, driving or using public transportation, shopping, walking $250 \mathrm{~m}$, cutting toenails and climbing stairs) and a participant's performance for each item was classified as independent, partially dependent or completely dependent. Those with one or more impaired ADL or IADL functions were defined as disabled. Frailty was assessed using a Frailty Index (FI), derived from the standard Comprehensive Geriatric Assessment (CGA) instrument (FI was primarily based on six domains: demographic characteristics, physical health, physical functions, living behaviour and social functions, mental health, and cognitive functions). ${ }^{27}$ Frailty was defined by a score of $\geq 0.25 .{ }^{28}$ Fractures were defined as a self-reported history of spontaneous fractures occurring in the past 2 years and falls were defined as those that occurred twice in the past year. Immobility was self-reported.

\section{Bias}

A door-to-door survey was conducted by formally trained interviewers using the CGA to ensure homogeneous reporting. Quality control procedures included regular field supervision and daily review of collected data. EpiData was used to establish the database, input and automatically verify the data.

\section{Study size}

The sample size was estimated to measure the prevalence of IC decline in older adults. Assuming an IC decline prevalence of approximately $30 \%$ with a precision margin of $2 \%$, the estimated sample size was 2016. Assuming a 15\% refusal or absence, we estimated a sample size adjusted to 2372 .

\section{Statistical methods}

All statistical analyses were performed using SPSS V.11.0. Count data were expressed as percentages. $\chi^{2}$ tests, t-tests and one-way analysis of variance were performed. Those with a p value less than 0.05 were included in the multivariable model. The weighted percentages were determined using the national standard population composition ratio based on the Sixth National Census (2010). A forward stepwise logistic regression was conducted to explore the association between the included factors and IC decline or between IC decline and adverse outcomes. A p value of $<0.05$ was considered statistically significant.

\section{Patient and public involvement}

Patients and the public were not involved in the planning and design of this study.

\section{RESULTS}

Of the total 5823 older adults, the average IC score was 9.14 \pm 1.304 , the median (IQR) score was 10 (1), and 2506 had IC decline, yielding a prevalence of $43.0 \%$ (weighted $39.9 \%)$. A total of $3317(57.0 \%), 1512(26.0 \%), 636$ (10.9\%), $271(4.7 \%), 75(1.3 \%)$ and $12(0.2 \%)$ participants showed decline in $0,1,2,3,4$ and 5 domains, respectively. The prevalence of IC decline was higher in women than in men $(43.0 \%$ vs $36.7 \%, \mathrm{p}=0.004)$. The prevalence of IC decline among older adults in rural areas was higher, by 1.36 times than in urban areas $(\mathrm{p}<0.001)$. The prevalence of IC decline increased with age, with the highest decline observed in individuals aged $\geq 80$ years $(66.6 \%)$ and the lowest in those aged $60-64$ years $(28.6 \% ; \mathrm{p}<0.001$; table 1). The number of participants with decline in the locomotion, cognition, vitality, sensory and psychological domains was 1039 (17.8\%), 646 (11.1\%), 735 (12.6\%), $824(14.2 \%)$ and $713(12.2 \%)$, respectively. For each domain, the prevalence was higher in women and those in rural areas, and increased with age, although there was no association between psychological domain and age (online supplemental table S2).

We observed differences in the prevalence of IC decline among the six regions: $57.7 \%$ in western China, $38.3 \%$ in northern China, 33.7\% in northwest China, 36.1\% in middle China, $16.9 \%$ in eastern China and $19.8 \%$ in northeast China (figure 1A). The prevalence of IC decline was higher in northern than southern regions $(46.9 \%$ vs $36.7 \%, \mathrm{p}<0.001$; figure $1 \mathrm{~B})$. The IC score decreased in older age groups $(9.53 \pm 0.88$ for the $60-64$-year age group, $49.37 \pm 1.06$ for the $65-69$-year age group, $9.18 \pm 1.22$ for the 70-74-year age group, $8.96 \pm 1.39$ for the $75-79$-year age group, $8.35 \pm 1.73$ for the 80 years and over age group $(\mathrm{p}<0.001$; figure 1C) $)$ and was higher in men than in women $(9.24 \pm 1.19$ vs $9.06 \pm 1.38, \mathrm{p}<0.001$; figure $1 \mathrm{D})$.

Next, we observed the prevalence of IC decline in different regions according to geography, sex and age. In urban areas, the weighted prevalence of IC decline differed across the six regions, with western urban regions showing the highest rates and eastern urban regions showing the lowest rates: $43.1 \%$ in western China, $36.1 \%$ in middle China, $33.7 \%$ in northwest China, $30.7 \%$ in northern China, $19.8 \%$ in northeast China and $11.8 \%$ in eastern China. Older adults living in rural areas included those residing in Beijing and Chengdu, and the prevalence of IC decline was higher in Chengdu than in Beijing ( $74.1 \%$ vs $47.2 \%, \mathrm{p}<0.001)$. The prevalence of IC decline among the six regions was different when analysed according to sex and age, with western regions showing the highest rate in both genders, in those aged $<75$ years and those aged $\geq 75$ years; the eastern regions showing the lowest rates in both genders and those aged $<75$ years; 
Table 1 Prevalence of intrinsic capacity decline in older adults by sex, area and age

Intrinsic capacity

decline Weighted Number of declined domains

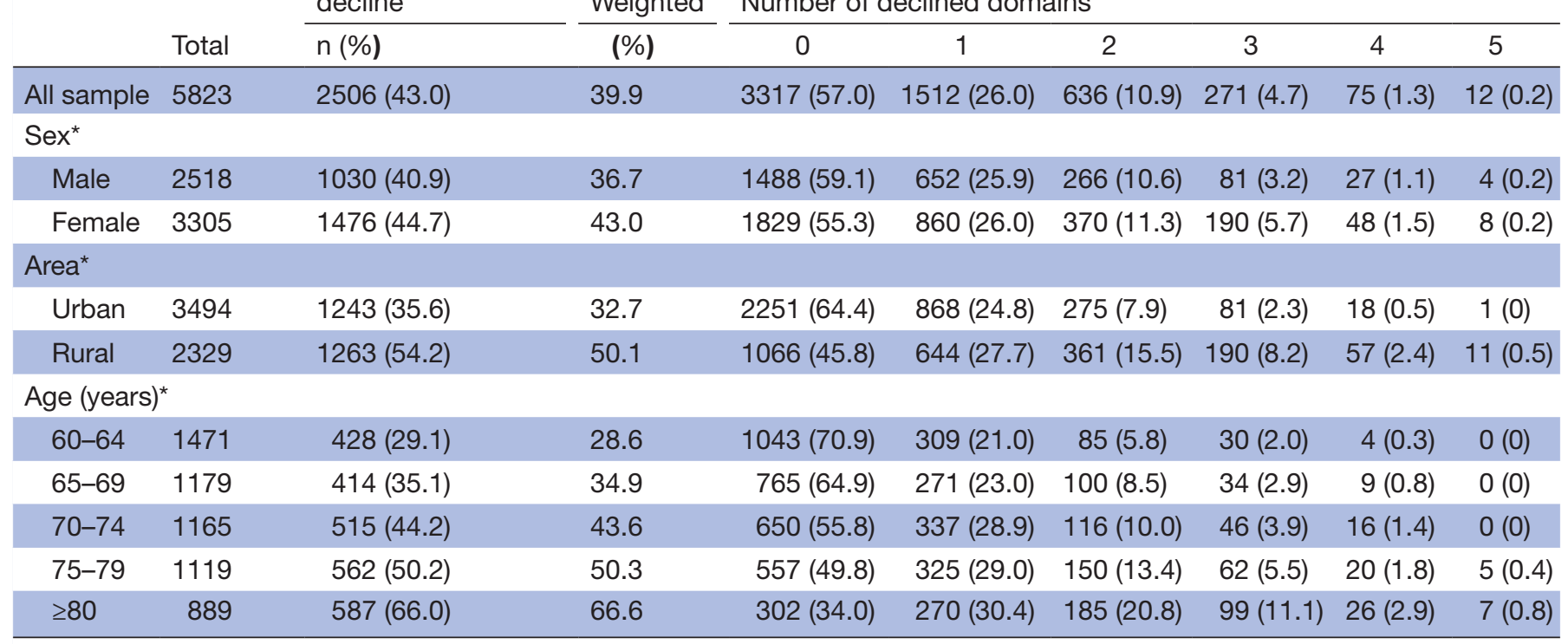

${ }^{*} \mathrm{P}<0.05$.

and east-north regions showing the lowest rates in those aged $\geq 75$ years $(\mathrm{p}<0.001$; figure 2$)$.

When comparing socio-psychological factors, chronic diseases and geriatric syndromes, a high prevalence of IC
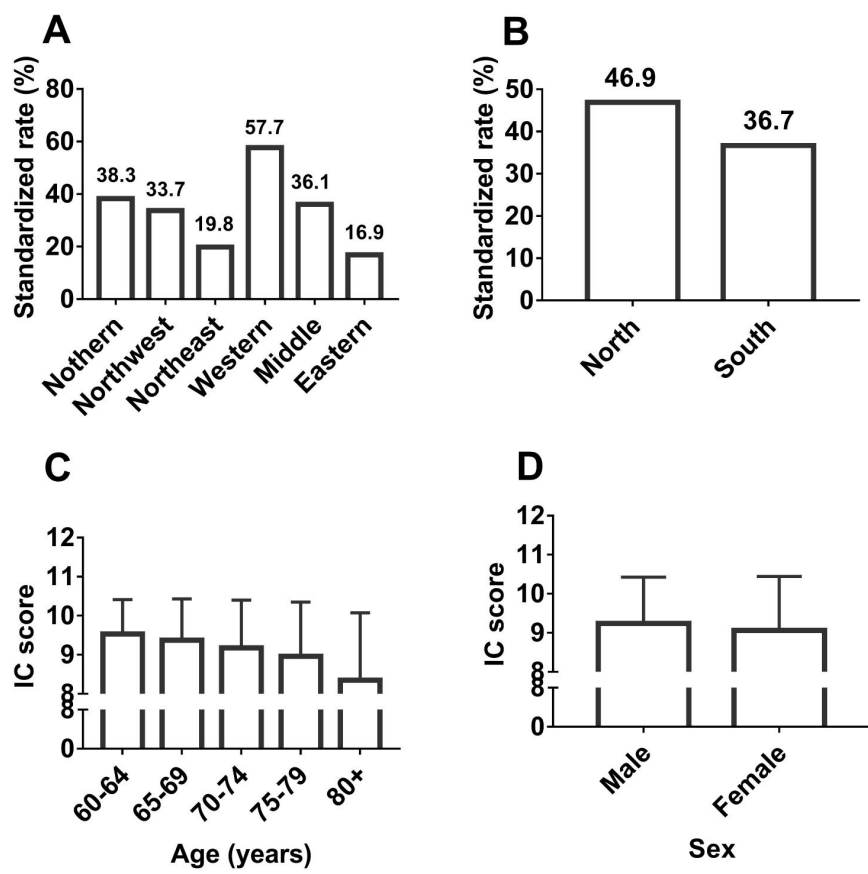

Figure 1 Intrinsic capacity in different regions, age and sex groups. (A) The comparison of the weighted prevalence of IC decline among the six regions of China $\left(\chi^{2}\right.$ test, $\left.p<0.01\right)$. (B) The comparison of the weighted prevalence of IC decline between north China and south China $\left(\chi^{2}\right.$ test, $\left.p<0.01\right)$. (C) The comparison of the IC score among different age groups (one-way ANOVA, $p<0.01$ ). (D) The comparison of the IC score between men and women (t-test, $p<0.01)$. ANOVA, analysis of variance; IC, intrinsic capacity. decline was observed in illiterate participants $(\mathrm{p}<0.001)$, those with low income $(\mathrm{p}<0.001)$, who were unmarried $(\mathrm{p}<0.001)$, did not exercise $(\mathrm{p}<0.001)$, consumed less meat diet $(\mathrm{p}<0.001)$ and consumed alcohol $(\mathrm{p}=0.043)$. Those with chronic diseases such as coronary heart disease $(p<0.001)$, stroke $(p<0.001)$, kidney disease $(\mathrm{p}=0.002)$, chronic obstructive pulmonary disease $(\mathrm{p}<0.001)$ and osteoarthritis $(\mathrm{p}<0.001)$, as well as geriatric syndromes $(\mathrm{p}<0.001)$ had a higher prevalence of IC decline, while there was a lower prevalence in older adults without diabetes $(\mathrm{p}=0.044$; online supplemental table S3). The prevalence of IC decline among individuals with $\geq 5$ chronic diseases was approximately two times higher than among individuals without chronic disease $(62.5 \%$ vs $30.9 \%, \mathrm{p}<0.001)$.

With IC decline as the dependent variable and the above factors as independent variables, forward logistic analysis showed that older age $(\mathrm{p}<0.001)$, northern region $(p<0.001)$, low education $(p<0.001)$, being unmarried $(p<0.001)$, low income $(p<0.001)$, less exercise $(p=0.003)$, less meat intake $(\mathrm{p}=0.003)$, insomnia $(\mathrm{p}<0.001)$, memory decline $(p<0.001)$, urinary incontinence $(p=0.001)$, constipation $(p=0.001)$, slowness $(p<0.001)$, chronic obstructive pulmonary disease $(\mathrm{p}<0.001)$ and osteoarthritis $(p<0.001)$ were independently associated with IC decline in older adults (table 2).

We further compared the frequency of adverse clinical outcomes between non-IC decline and IC decline groups. Participants with IC decline were much more likely to be frail (22.2\% vs $1.2 \%, \mathrm{p}<0.001)$, disabled $(15.0 \%$ vs $1.0 \%$, $\mathrm{p}<0.001)$ and have falls $(7.9 \%$ vs $1.8 \%, \mathrm{p}<0.001)$, fractures $(4.7 \%$ vs $2.2 \%, \mathrm{p}<0.001)$ and be immobile $(20.4 \%$ vs $3.4 \%, \mathrm{p}<0.001)$. Logistic regression showed that, after adjustment for age, sex, area, district, marriage, 

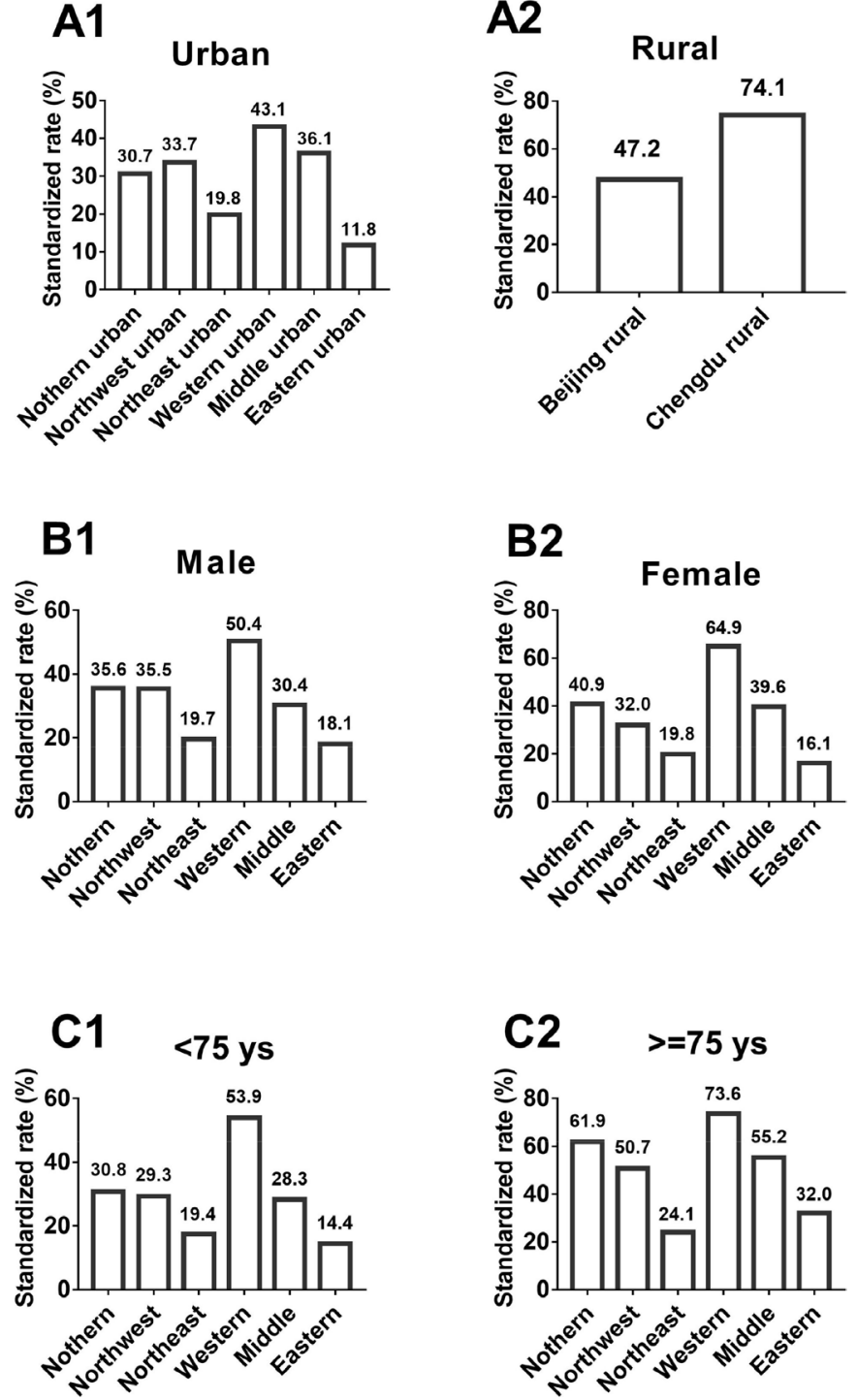

Figure 2 The weighted prevalence of declines in intrinsic capacity in community-dwelling older adults living in the urban area (A1), rural area (A2), men (B1), women (B2), $<75$ ys (C1) and $\geq 75$ ys (C2). All were analysed using the $\chi^{2}$ test, $p<0.01$. Ys, years.

education, waist-hip ratio, smoking, alcohol consumption, exercise, income and chronic diseases, IC decline was independently associated with risks of frailty (adjusted $\mathrm{OR}=19.021, \mathrm{p}<0.001$ ), disability (adjusted $\mathrm{OR}=8.611$, $\mathrm{p}<0.001$ ), falls (adjusted $\mathrm{OR}=3.053$, $\mathrm{p}<0.001$ ), fractures (adjusted $\mathrm{OR}=1.656, \mathrm{p}=0.003$ ), and immobility (adjusted $\mathrm{OR}=4.403, \mathrm{p}<0.001 ;$ table 3$)$.

\section{DISCUSSION}

In this nationally representative population-based crosssectional study, the weighted prevalence of IC decline was as high as $39.9 \%$ in community-living Chinese older adults aged $\geq 60$ years, which is approximately five times higher than the prevalence of frailty in the same population. ${ }^{29}$ The frequency of participants with a decline in IC domains varied from $11.1 \%$ (cognition) to $17.8 \%$ (locomotion). These findings highlight the need to focus on functional trajectories rather than the traditional disease-centred approach. However, well-established concepts such as frailty (which is related as an increasingly problematic consequence of population ageing) threaten the sustainability of healthcare resources as most people seek healthcare attention only when they are at their worst health state. Furthermore, studies have reported that current integrated care models have not significantly reduced healthcare utilisation nor consistently reduced mortality. ${ }^{30}$ Thus, healthcare model such as ICOPE with IC as its core may support the modernisation of current healthcare systems and at the same time be more personalised. Results of ongoing INStitute for Prevention healthy agIng and medicine REjuvenative (INSPIRE) study conducted in Europe which is based on the ICOPE model ${ }^{31}$ will have strong implications for the effectiveness of the IC approach.

Our study demonstrated that the prevalence of IC decline among participants was higher in northern than southern regions of China. Furthermore, there were differences in the prevalence of IC decline among the six regions, which was consistent with previous studies in which the incidence of frailty ${ }^{32}$ and prevalence of disability ${ }^{20}$ were more common in the north than the south, indicating the importance of implementing the ICOPE protocol, especially in northern China. We found that the IC score decreased in older age groups, while longitudinal studies showed that the effect of age on the incidence of ADL dependency was modified by IC. ${ }^{15}$

Logistic regression showed that factors such as age, region, low education, unmarried status, low income, less exercise, less meat intake, insomnia, memory loss, urinary incontinence, constipation, slowness, chronic obstructive pulmonary disease and osteoarthritis were independently associated with IC decline in older adults, demonstrating that efforts to develop strategies and health policies to identify and manage modifiable variables are urgently required. A longitudinal study in New Zealand showed that neighbourhood environments and IC interact to affect the quality of life in older persons ${ }^{33}$ Since the above factors are independently associated with an individual's IC, multidomain and complex interventions provide a better option for the prevention and management of IC decline. Accordingly, the WHO recommends screening using instruments such as the ICOPE screening tool as the first step at the primary care level followed by a suitable care plan. ${ }^{334}$

We demonstrated that after adjusting for sociodemographic variables, age-related factors and chronic conditions, IC decline was independently associated with risk of frailty, disability, falls, fractures and immobility, which is in line with the finding that IC predicted the incidence of loss of ADLs and IADLs, ${ }^{15}$ and further indicates that evaluations of IC implementation may be extremely important to avoid further deterioration (which could be a severe frailty state). The term frailty refers to health deficits of an ageing individual while IC emphasises on 
Table 2 Stepwise forward logistic regression for associated factors with intrinsic capacity decline

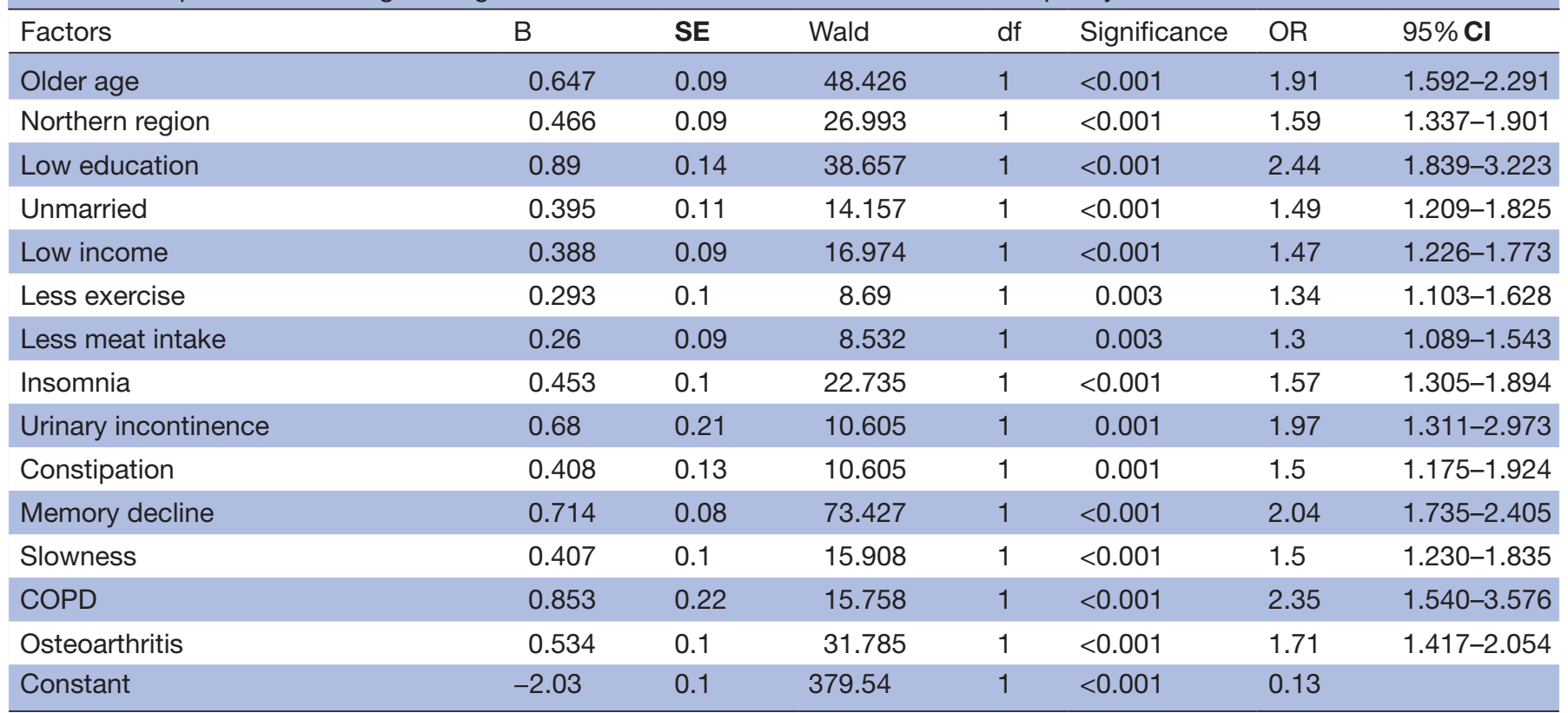

The variables not in the equation were sex, living areas, alcohol consumption, coronary heart disease, diabetes, stroke and kidney disease. COPD, chronic obstructive pulmonary disease; df, degrees of freedom.

positive health aspects; therefore, IC may be considered as an evolution of frailty in certain respects. ${ }^{35}$ It is also noteworthy that the World Report on Ageing and Health proposes the concept of IC as central for healthy ageing $^{4}$; thus, the concept of IC could serve as a bridge between geroscience and healthy ageing. ${ }^{14}$ Furthermore, an IC approach has the benefit of tracing trajectories for progression to adverse clinical outcomes such as frailty and disability, and testing the effectiveness of interventions implemented at the individual level. Thus, an IC model is of great significance for facing the unmet needs of older adults.

This study has several limitations. First, the primary limitation is being unable to establish causality due to the cross-sectional design. Further longitudinal studies with a larger sample size are urgently required. Second, vitality was defined by BMI instead of specific nutritional assessment, which may have affected some of the results. Since most of the diabetic patients in our study had a higher BMI, this could explain the lower IC decline in participants with diabetes. Third, we used a composite total score instead of a weighted score, so further statistical approaches should be conducted to compute IC scores. Fourth, self-reporting for hearing impairment may misestimate hearing loss (although WHO recommends whisper test for assessing hearing loss). Fifth, 1040 participants who were unable to complete the IC assessment were excluded in this study. The excluded participants who were older and frailer, had more chronic diseases, and lower scores in each domain (online supplemental table $\mathrm{S} 1$ ), which may have misestimated the prevalence of IC decline.

To the best of our knowledge, this study is the first nationally representative large sample of population-based

Table 3 Multivariate logistic regression analysis for intrinsic capacity decline associated with risk of adverse clinical outcomes

\begin{tabular}{|c|c|c|c|c|c|c|}
\hline & \multicolumn{3}{|c|}{ Model 1} & \multicolumn{3}{|l|}{ Model 2} \\
\hline & OR & $95 \% \mathrm{Cl}$ & $P$ value & OR & $95 \% \mathbf{C l}$ & $P$ value \\
\hline Frailty & 19.625 & $14.044-27.423$ & $<0.001$ & 19.021 & $12.882-28.084$ & $<0.001$ \\
\hline Fall & 3.671 & $2.699-4.993$ & $<0.001$ & 3.053 & $2.232-4.177$ & $<0.001$ \\
\hline Fracture & 1.965 & $1.448-2.666$ & $<0.001$ & 1.656 & $1.195-2.295$ & 0.003 \\
\hline
\end{tabular}

Model 1: adjusted by age, sex, area and district.

Model 2: adjusted by age, sex, area, district, marriage, education, waist-hip ratio, smoking, alcohol consuming, exercise, income and chronic diseases.

Reference: non-intrinsic capacity decline. 
older people focusing on IC in China. Most of the components of IC were assessed using unified measured performance tests, thus avoiding response or interviewer bias. Standardised protocols, as well as regularly and randomly performed internal quality checks of the data, were used to avoid the quality disadvantage of the multicentre design.

In conclusion, this study provided a preliminary understanding of the IC status of Chinese older adults. Our results indicate that efforts promoting IC to delay functional dependence should focus on negative social factors, poor lifestyle, chronic diseases and geriatric syndromes. This study also validated the IC concept in Chinese population. In fact, China has already implemented the ICOPE approach into clinical practice and launched a pilot multicentre study called 'China Ageing, Resilience and Intrinsic Capacity Study (CARICS)' to identify and manage IC decline to improve wellbeing among community-living older adults. We hope that this approach of IC will be a fundamental tool towards healthy ageing worldwide.

Acknowledgements We acknowledge all the people who participated in the China Comprehensive Geriatric Assessment Study from the following hospitals: Xuanwu Hospital, Beijing Hospital, Beijing Institute of Geriatrics, General Hospital of PLA, Peking University Third Hospital, The First Affiliated Hospital of Xi'an Jiaotong University, Xijing Hospital of The Fourth Military Medical University, The First Affiliated Hospital of Harbin Medical University, West China Hospital of Sichuan University, First Affiliated Hospital of Chongqing Medical University, Xiangya Hospital of Central South University, The Third Xiangya Hospital of Central South University and Ruijin Hospital of Shanghai Jiao Tong University School of Medicine.

Contributors LM and ZT conceived of the research, reviewed related literature, oversaw analysis and was responsible for the final drafting of the paper. JKC, FS, LZ and YL contributed to conceptualisation, reviews of related literature and drafting of the paper. All authors reviewed and approved the final manuscript submitted for publication.

Funding This work was supported by the following grants: National Key R\&D Program of China (2020YFC2008604), the Natural Science Foundation of Beijing (7202059), Beijing Municipal Hospital Scientific Research Training Project (PX2020036), Beijing Municipal Health Commission (Jing2019-2) and Milstein Medical Asian American Partnership Foundation Project Award in Geriatrics.

Competing interests None declared.

Patient consent for publication Not required.

Ethics approval All study participants provided informed consent, and the study design was approved by the ethics review board of Xuanwu Hospital, Capital Medical University.

Provenance and peer review Not commissioned; externally peer reviewed.

Data availability statement No data are available. No additional data are available.

Supplemental material This content has been supplied by the author(s). It has not been vetted by BMJ Publishing Group Limited (BMJ) and may not have been peer-reviewed. Any opinions or recommendations discussed are solely those of the author(s) and are not endorsed by BMJ. BMJ disclaims all liability and responsibility arising from any reliance placed on the content. Where the content includes any translated material, BMJ does not warrant the accuracy and reliability of the translations (including but not limited to local regulations, clinical guidelines, terminology, drug names and drug dosages), and is not responsible for any error and/or omissions arising from translation and adaptation or otherwise.

Open access This is an open access article distributed in accordance with the Creative Commons Attribution Non Commercial (CC BY-NC 4.0) license, which permits others to distribute, remix, adapt, build upon this work non-commercially, and license their derivative works on different terms, provided the original work is properly cited, appropriate credit is given, any changes made indicated, and the use is non-commercial. See: http://creativecommons.org/licenses/by-nc/4.0/.

ORCID iD

Lina Ma http://orcid.org/0000-0001-7630-6960

\section{REFERENCES}

1 Prince MJ, Wu F, Guo Y, et al. The burden of disease in older people and implications for health policy and practice. Lancet 2015;385:549-62.

2 Stoop A, Lette M, Ambugo EA, et al. Improving Person-Centredness in integrated care for older people: experiences from thirteen integrated care sites in Europe. Int J Integr Care 2020;20:16 https:// www.ijic.org/article/10.5334/ijic.5427/

3 Integrated care for older people: guidelines on community-level interventions to manage declines in intrinsic capacity [online], 2017. Available: https://pubmed.ncbi.nlm.nih.gov/29608259/ [Accessed 3 Jul 2020].

4 World Health Organization. Ageing and health [online], 2018. Available: http://www.who.int/news-room/fact-sheets/detail/ageingand-health [Accessed 23 Jun 2018].

5 Yu R, Cecilia Tong JW. Effect of an integrated care model for pre-frail and frail older people living in community. Age Ageing [online] 2020 https://academic.oup.com/ageing/article-abstract/doi/10.1093/ ageing/afaa087/5848668? redirectedFrom=fulltext

6 Framework implementation of the INSPIRE ICOPE-CARE program in collaboration with the world Health organization (who) in the Occitania region. J Frailty Aging 2020:1-7.

7 Charles A, Buckinx F, Locquet M. Prediction of adverse outcomes in nursing home residents according to intrinsic capacity proposed by the world Health organization | the journals of gerontology: series a | Oxford academic. J Gerontol A Biol Sci Med Sci 2019.

8 Giudici KV, de Souto Barreto P, Guerville F, et al. Associations of C-reactive protein and homocysteine concentrations with the impairment of intrinsic capacity domains over a 5-year follow-up among community-dwelling older adults at risk of cognitive decline (MAPT study). Exp Gerontol 2019;127:110716.

9 Daskalopoulou C, Chua K-C, Koukounari A, et al. Development of a healthy ageing index in Latin American countries - a 10/66 dementia research group population-based study. BMC Med Res Methodol 2019;19:226.

10 Giudici KV, de Souto Barreto P, Beard J, et al. Effect of long-term omega-3 supplementation and a lifestyle multidomain intervention on intrinsic capacity among community-dwelling older adults: secondary analysis of a randomized, placebo-controlled trial (MAPT study). Maturitas 2020;141:39-45.

11 Huang $\mathrm{CH}$, Umegaki $\mathrm{H}$, Makino T. Effect of various exercises on intrinsic capacity in older adults with subjective cognitive concerns. J Am Med Dir Assoc 2020

12 Gutiérrez-Robledo LM, García-Chanes RE, Pérez-Zepeda MU. Allostatic load as a biological substrate to intrinsic capacity: a secondary analysis of CRELES. J Nutr Health Aging 2019;23:788-95.

13 Ma L, Liu P, Zhang Y. High serum tumor necrosis factor receptor 1 levels are related to risk of low intrinsic capacity in elderly adults. $J$ Nutr Heal Aging 2020:1-3.

14 Barreto P de S, Guyonnet S, Ader I. The Inspire research Initiative: a program for Geroscience and healthy aging research going from animal models to humans and the healthcare system. J Frailty Aging 2020:1-8.

15 Beard JR, Jotheeswaran AT, Cesari M, et al. The structure and predictive value of intrinsic capacity in a longitudinal study of ageing. BMJ Open 2019;9:e026119.

16 Ramírez-Vélez R, Correa-Bautista JE, García-Hermoso A, et al. Reference values for handgrip strength and their association with intrinsic capacity domains among older adults. $J$ Cachexia Sarcopenia Muscle 2019;10:278-86.

17 Cesari M, Araujo de Carvalho I, Amuthavalli Thiyagarajan J, et al. Evidence for the domains supporting the construct of intrinsic capacity. J Gerontol A Biol Sci Med Sci 2018;73:1653-60.

18 Nestola T, Orlandini L, Beard JR, et al. COVID-19 and intrinsic capacity. J Nutr Health Aging 2020;24:1-4.

19 Ma L, Zhang L, Zhang Y, et al. Cognitive frailty in China: results from China comprehensive geriatric assessment study. Front Med 2017;4:174.

20 Ma L, Li Z, Tang Z, et al. Prevalence and socio-demographic characteristics of disability in older adults in China: findings from China comprehensive geriatric assessment study. Arch Gerontol Geriatr 2017;73:199-203. 
21 Ma L, Tang Z, Zhang L, et al. Prevalence of frailty and associated factors in the community-dwelling population of China. J Am Geriatr Soc 2017.

22 WHO. Meeting of the clinical consortium on healthy ageing. WHO, 2018.

23 Guralnik JM, Simonsick EM, Ferrucci L, et al. A short physical performance battery assessing lower extremity function: association with self-reported disability and prediction of mortality and nursing home admission. J Gerontol 1994;49:M85-94.

$24 \mathrm{Li} \mathrm{H}$, Jia J, Yang Z. Mini-Mental state examination in elderly Chinese: a population-based normative study. J Alzheimers Dis 2016;53:487-96

25 Screening for malnutrition: a multidisciplinary responsibility. Development and Use of the Malnutrition Universal Screening Tool ('MUST') for adults. Redditch: BAPEN 2003.

26 Chan AC. Clinical validation of the geriatric depression scale (GDS): Chinese version. J Aging Health 1996;8:238-53.

$27 \mathrm{Ma} \mathrm{L}$, Zhang L, Tang Z, et al. Use of the frailty index in evaluating the prognosis of older people in Beijing: a cohort study with an 8-year follow-up. Arch Gerontol Geriatr 2016;64:172-7.

28 Rockwood K, Andrew M, Mitnitski A. A comparison of two approaches to measuring frailty in elderly people. J Gerontol A Biol Sci Med Sci 2007;62:738-43.
29 Ma L, Tang Z, Zhang L, et al. Prevalence of frailty and associated factors in the community-dwelling population of China. J Am Geriatr Soc 2018;66:559-64.

30 Kasteridis P, Mason A, Street A. Evaluating integrated care for people with complex needs. J Health Serv Res Policy 2021;26:46-53.

31 Takeda C, Guyonnet S, Sumi Y, et al. Integrated care for older people and the implementation in the INSPIRE care cohort. J Prev Alzheimers Dis 2020;7:70-4.

$32 \mathrm{Xu} \mathrm{W}, \mathrm{Li}$ Y-X, Wu C. Incidence of frailty among community-dwelling older adults: a nationally representative profile in China. BMC Geriatr 2019;19:378.

33 Stephens C, Allen J, Keating N, et al. Neighborhood environments and intrinsic capacity interact to affect the health-related quality of life of older people in New Zealand. Maturitas 2020;139:1-5.

$34 \mathrm{WHO}$. Integrated care for older people (ICOPE): guidance for personcentred assessment and pathways in primary care. who, 2019. Available: http://www.who.int/ageing/publications/icope-handbook/ en/ [Accessed 3 Jul 2020].

35 Belloni G, Cesari M. Frailty and intrinsic capacity: two distinct but related constructs. Front Med 2019;6:133. 